\title{
Kernos
}

Revue internationale et pluridisciplinaire de religion grecque antique

3 | 1990

Varia

\section{Le paradoxe étrusque : une parole inspirée sans oracles prophétiques}

\section{Dominique Briquel}

\section{OpenEdition \\ Journals}

\section{Édition électronique}

URL : http://journals.openedition.org/kernos/971

DOI : 10.4000/kernos.971

ISSN : 2034-7871

\section{Éditeur}

Centre international d'étude de la religion grecque antique

\section{Édition imprimée}

Date de publication : 1 janvier 1990

ISSN : 0776-3824

\section{Référence électronique}

Dominique Briquel, «Le paradoxe étrusque : une parole inspirée sans oracles prophétiques », Kernos [En ligne], 3 | 1990, mis en ligne le 19 avril 2011, consulté le 01 mai 2019. URL : http:// journals.openedition.org/kernos/971; DOI : 10.4000/kernos.971 


\section{LE PARADOXE ÉTRUSQUE : UNE PAROLE INSPIRÉE SANS ORACLES PROPHÉTIQUES}

Les auteurs qui ont parlé de la religion étrusque ont souvent employé à son propos le terme de "religion révélée». C'est ainsi qu'on trouve, dans l'ouvrage classique qu'est La vie quotidienne chez les $\hat{E}$ trusques de J. Heurgon, cette formule : "la religion étrusque, à la différence des religions grecque et romaine, mais à la ressemblance des religions juive et chrétienne, était une religion révélée»1. Et on pourrait citer des remarques analogues sous la plume, entre autres, de R. Bloch, A. Hus, G. Dumézil, ou encore O. von Vacano, M. Pallottino, A.-J. Pfiffig 2 . Les croyances des Étrusques étaient en effet présentées comme étant le résultat d'une révélation, de la parole inspirée de prophètes qui avaient transmis des vérités d'origine divine aux hommes. C'est dire qu'on peut tout aussi bien présenter la religion étrusque comme une "religion de la parole», et dire qu'elle repose sur l'idée d'une communication orale aux hommes de vérités révélées par la divinité par l'intermédiaire de ces êtres privilégiés que sont les prophètes.

Rappelons les faits. Les Étrusques connaissaient plusieurs figures de prophètes, ancrées dans la tradition locale ${ }^{3}$, dont au moins deux nous

1 Voir dans La vie quotidienne chez les Étrusques, Paris, 1957, p. 283-286.

2 Voir respectivement Les Étrusques, Paris, 1954, p. 93-94; Les Étrusques, peuple secret, Paris, 1957, p. 183-184; Religion romaine archaïque, Paris, 1966, appendice La religion des Étrusques, p. 601-602; Die Etrusker in der Welt der Antike, Hambourg, 1957, p. 45; Etruscologia 6 , Milan, 1973, p. 272-273; Religio Etrusca, Graz, 1979, p. 37.

3 Nous mettons à part d'autres figures de prophètes, parfois regroupées avec Tagès et Vegoia, mais qu'il nous paraît préférable de tenir distinctes. Ainsi le Cacu qui apparaft sur plusieurs miroirs et urnes étrusques, dans une scène où on le voit assailli, au moment où il prophétise, par ces condottieri que sont les frères Vibenna (pour les données, voir en part. J. HEURGoN, Vie quotidienne, p. 282-284). Mais F.-H. MAssa-PaIrault nous semble avoir eu raison d'expliquer cette scène à la lumière des traditions grecques sur la capture d'Hélénos par Ulysse et Diomède, condition nécessaire à la prise de Troie (Recherches sur l'art et l'artisanat étrusco-italiques à l'époque hellénistique, Rome, 1985, p. 47-57) : il s'agirait alors d'un motif lié aux luttes entre Rome, conçue comme une nouvelle Troie, et les Étrusques, qui se considèrent comme les heritiers des Grecs qui ont pris Troie, et cette histoire ne concernerait pas directement le prophétisme toscan. De même F.-H. Massa-PairaulT (communication au IV Convegno Internazionale della Fondazione per il Museo 
sont saisissables ${ }^{4}$, l'enfant miraculeux Tagès, surgi du sillon d'un champ qu'un paysan labourait près de Taquinia ${ }^{5}$, et la nymphe Vegoia, qui, telle qu'elle est connue par la tradition littéraire ${ }^{6}$, doit prolonger une figure de $\mathrm{Lasa}^{7}$ - équivalent étrusque de «nymphe» - liée à la

C. Faina, Orvieto, 1987, à paraître dans les Actes) nous semble avoir justement refusé de voir dans la mention chez Fulgence, à propos d'Etrusca disciplina, de Tageticis et Bacitidis voluminibus (Serm. ant., 4) une allusion, outre à Tagès, à Vegoia, dont le nom aurait été altéré (dans ce sens p. ex. K.O. MÜLLER, W. DEECKE, Die Etrusker ${ }^{2}$, II, Stuttgart, 1877, p. 30-31, avec n. 45), ni une référence imaginaire (C.O. THULIN, Die etruskiche Disciplin, I, Göteborg, 1906, p. 2); elle a proposé d'y reconnaître une allusion à un Bacis, soit à l'un des devins grecs - et non étrusques - portant ce nom (voir A. BOUCHE-LECLECQ, Histoire de la divination dans l'antiquité, II, Paris, 1880, p. 105-107). Il s'agit donc d'une référence à une figure de devin grec, qui a été mis en rapport avec la divination étrusque (de même que l'ont parfois été Calchas, Tirésias, Pollès, voir notre art. Divination étrusque et mantique grecque : la recherche d'une origine grecque de l'Etrusca disciplina, à paraître). Restent également extérieurs à la réalité du prophétisme étrusque tant bien sûr la tête vaticinante d'Orphée qui figure sur plusieurs miroirs toscans que la figure, très discutée, également connue par des miroirs, d'Aliunea (dont D. Rebuffat-Emmanuel, Aliunea n'a jamais existé, in Latomus, 43 [1984], p. 501-17, expliquait même le nom comme une déformation due à un graveur illettré de celui de Palamède), qu'une proposition suggestive de A. MAGGIANI rapproche maintenant de l'Albunea que fait intervenir Virgile dans l'Éneide, et rapporte donc au milieu latin de même que le Faun / Phaun qui serait à mettre en relation avec Faunus (La divination oraculaire en Étrurie, in La divination dans le monde-étrusco-italique, III, Caesarodunum, Suppl. 56 [1986], p. 6-48).

4 Assurément d'autres figures ont pu exister que celles qui nous sont connues. Nous rappellerons simplement que l'inscription $C I L, \mathrm{XI}, 3370$, où E. BORMANN proposait des restitutions amenant à y voir la mention d'une révélation dont aurait été bénéficiaire le célèbre haruspice du Ier s. av. J.-C. Tarquitius Priscus (Arch. Epigr. Mittheil. aus Öster.-Ungarn [1887], p. 14 sq.; dans ce sens encore J. Heurgon, Tarquitius Priscus et l'organisation de l'ordre des haruspices sous l'empereur Claude, in Latomus, 12 [1953], p. 402-417), a fait l'objet d'un réexamen de M. ToRelli, Elogia Tarquiniensia, Rome, 1975, p. 105-116, qui élimine tant le nom de Tarquitius que l'idée d'une révélation.

5 Les données sont commodément rassemblées dans l'article (par ailleurs contestable) de J. R. WooD, The Myth of Tages, in Latomus, 39 (1980), p. 4-20.

6 Les références sont relativement limitées : SERV., ad Verg., Aen., VI, 72 (avec la forme Begoe du nom), AMM. MARC., XVII, 10, 2 (pour des libri Vegonici). Mais on dispose d'une prophétie présentée comme émanant de Vegoia (ap. Gromm. Vet., éd. Lachmann, 350-351; sur ce texte, en dernier lieu A. VALVo, Termini moti, domini e servi in Etruria nel I sec. a. C., alcune considerazioni intorno alla cosiddetta «profezia di Vegoia», in Athenaeum, 65 [1987], p. 427-451).

7 Sur cette notion, L. RALLo, Lasa, iconografia e esegesi, Rome, 1975. 
région de Chiusi, la Lasa Vecu ou Vecuvia ${ }^{8}$. La distinction entre ces diverses figures semble en effet avoir été due, au moins au départ, au caractère strictement local de ces prophètes. Cela est patent pour Tagès. $\mathrm{Sa}$ légende le fait apparaître à Tarquinia, et il semble que nous puissions repérer le lieu d'où il aurait surgi du sol, dans l'ensemble du temple de l'Ara della Regina, à la périphérie de la cité ${ }^{9}$ : c'est au moins ainsi que l'on est tenté d'interpréter la présence d'un petit édifice rectangulaire au sein même du podium du temple. Mais que les diverses cités qui composaient la dodécapole étrusque aient voulu mettre en avant leur prophète local n'est pas pour surprendre dans un monde pour lequel on a souvent souligné la désunion entre les cités, leurs rivalités ${ }^{10}$ - qui n'avaient rien à envier à ce qu'on constate en Grèce ! Ce qui est en tous cas significatif du point de vue des conceptions religieuses est que les diverses cités développent des légendes parallèles, mettent en scène chaque fois leur propre prophète - en tendant à lui attribuer une valeur panétrusque : ainsi Tagès, le prophète de Tarquinia, est censé enseigner chez Cicéron à toute l'Étrurie, venue en foule profiter de ses leçons ${ }^{11}$. C'est dire l'importance de la parole inspirée aux yeux des Étrusques : elle est un moyen privilégié pour les hommes d'entrer en communication avec les dieux, de recevoir des indications sur leur volonté.

Voici donc la situation pour la légende : le prophétisme y occupe sans conteste une place remarquable. Mais si nous nous tournons en revanche vers la réalité des pratiques concrètes, les choses sont fort

8 Le personnage est connu par une représentation sur un miroir, avec le nom de Lasa Vecuvia (E. GERHARD, Etruskische Spiegel, III, Leipzig-Berlin, 1863, p. 2223, I, pl. 37; A.RALlo, op. cit. n. 7, p. 32-35, pl. 191-192) et une gemme en or de Todi, avec la forme Lasa Vecu (M.A. RIzzo, in L'oro degli Etruschi, Rome, 1983, p. 215, fig. 226, 309). Le nom est attesté dans la région de Chiusi (mais voir aussi, pour le caractère volsinien de son interlocuteur Arruns Velthymnus, G. ColonNa, Società et cultura a Volsinii, in Volsinii e la dedecapoli etrusca, Atti del II Convegno della Fondazione per il Museo C. Faina, Orvieto, 1983 [1985], p. 112). Sur la question, en dernier lieu F.-H. Massa-Pairault, Lasa Vecu, Lasa Vecuvia, in DArch, 3, 6 (1988), p. 133-43.

9 Voir M. TORELLI, Storia degli Etruschi, Rome-Bari, 1982, p. 169; Studi in onore di T. Dhorn, Rome, 1982, p. 122-123; F.-H. MASSA-PAIRAulT, La divination en Étrurie, le IVe s., période critique, in La divination dans le monde étruscoitalique, Caesarodunum, Suppl. 52 (1985), p. 71-73.

10 Nous citerons simplement l'article classique de L. PARETI, La disunione politica degli Etruschi e $i$ suoi riflessi storici ed archeologici, in RPAA, 7 (1929-1930), p. 89-100.

11 CiC., De div., II, 23 (50) : ... concursum esse factum, totamque brevi tempore in eum locum Etruriam convenisse. Tum illum plura locutum multis audientibus... Cicéron est pour nous le plus ancien témoin de la légende. 
différentes. On ne peut apparemment pas affirmer l'existence d'une utilisation de cette parole inspirée au niveau des pratiques divinatoires.

Et pourtant il est certain que la divination est un aspect essentiel de la civilisation, de la mentalité étrusques. L'homme étrusque vit dans un monde où tout lui semble commandé par un destin voulu par les dieux, sinon par des puissances mystérieuses s'imposant aux dieux euxmêmes, tels les dii consentes ou complices, ou encore plus les dii superiores et involuti qui pèsent sur les décisions de Jupiter lui-même ${ }^{12}$. Comme l'atteste un document exceptionnel, le foie de bronze découvert à Plaisance qui montre la reproduction sur cette partie centrale et privilégiée de l'animal sacrifié du partage du ciel entre les divers dieux ${ }^{13}$, la doctrine étrusque, telle que l'enseignaient et la pratiquaient les haruspices, établit un rapport direct entre le monde où nous vivons et celui des dieux : dans ces conditions, il est essentiel que la communication soit établie entre les deux, que les hommes sachent ce que les dieux attendent d'eux et leur promettent. Et c'est la divination qui établit cette communication. Aussi la doctrine religieuse étrusque, l'Etrusca disciplina, qui eut tellement d'importance aux yeux des Romains que, vainqueurs de l'Étrurie, ils n'en donnèrent pas moins un rôle officiel dans leurs procédures religieuses aux devins de la nation vaincue ${ }^{14}$, est-elle avant tout, outre une doctrine des rites, une science de la divination ${ }^{15}$.

Mais parmi les techniques divinatoires ainsi mises en œuvre, il n'y a pas de place pour la divination inspirée, la parole du prophète exprimant directement ce que lui insuffle la divinité. La divination étrusque est une science des signes, fondée sur l'observation et l'interprétation de phénomènes extérieurs à l'homme ${ }^{16}$. Elle est exercée par des spécialistes, les haruspices, qui ne sont en rien des prophètes, mais doivent leur nom à une de ces techniques, l'hépatoscopie, fondée sur l'examen du foie des victimes sacrificielles. Mais leur art ne se

12 Sur ces notions, voir principalement SEN., N.Q., II, 47. Pour l'ensemble des données, C.O. THULIN, op. cit. n. 3, I, p. 181-183. Sur la question en général, voir les ouvrages cités aux n. 1 et 2.

13 Sur cette question, en dernier lieu A. MAGGIANI, Qualche osservazione sul fegato di Piacenza, in $S E$, 50 (1984), p. 54-88.

14 Sur ce point, voir maintenant B. MAC BAIN, Prodigy and Expiation, Bruxelles, 1984.

15 L'Etrusca disciplina était classiquement répartie entre la brontoscopie, l'hépatoscopie et le rituel (Cf. CIC., De div., I, 33, distinguant dans le corpus des livres sacrés étrusques les libri fulgurales, haruspicini et rituales).

16 Pour l'ensemble des données, C.O. THulin, Die etruskische Disciplin, Göteborg, 1906-1909. 
limitait pas à cela : ils étaient aussi particulièrement versés dans l'observation des foudres, ou brontoscopie. Et plus généralement, des signes aussi divers que les tremblements de terre, la croissance anormale d'un arbre, le vol et le chant des oiseaux relevaient de leur compétence.

Ces haruspices opéraient à la demande - à la manière du fameux Spurinna, haruspice attitré de César ${ }^{17}$, ou, à un niveau plus modeste, des haruspices de bas étage dont se moquait Caton ${ }^{18}$. Mais il a existé aussi en Étrurie un culte oraculaire - et qui, lui non plus, ne semble pas avoir pris la forme d'une consultation de prophètes inspirés ${ }^{19}$. A. Maggiani a repris récemment l'ensemble des données fournies par l'archéologie ${ }^{20}$. Nous pouvons reprendre la liste qu'il donne : «un modèle de coffret (boîte à sortes) de Véies, une sors en bronze de Viterbe, une en bronze d'Arezzo, avec le nom du dieu Suris, identifiable avec Apollon, un caillou portant le nom d'Aplu, soit Apollon, toujours d'Arezzo" 21 . On le voit, on a la trace de la pratique de la cléromancie, ou d'une lithomancie de forme analogue. Mais on n'a pas d'indices du recours à l'inspiration directe, l'enthousiasme mantique; sur ce point la conclusion de C.O. Thulin au début de ce siècle reste valable 22 .

17 Sur le personnage, M. TORELLI, Elogia Tarquiniensia, p. 105-135; E. RAWson, Caesar, Etruria and the Disciplina Etrusca, in JRS, 68 (1978), p. 132-152.

18 Voir CIC., De div., II, 14 (51) : vetus autem illud Catonis admodum scitum est, qui mirari se aiebat quod non rideret haruspex haruspicem cum vidisset.

19 La mention dans une version ancienne de la légende de Romulus (Promathion, ap. Plut., Rom., 2, $1=817$ F 1 J.) d'un oracle de Téthys en Étrurie qui aurait prédit au roi d'Albe Tarchétios que du phallus surgi de son foyer naîtrait un fils exceptionnel n'impose pas nécessairement l'idée d'une procédure de type prophétique. Une divination par les sortes, effectivement attestée pour l'Etrurie, est envisageable. Sur la localisation de cet oracle, R.H. KLAusEn, Aeneas und die Penaten, II, Leipzig, 1890, p. 722; J. HeURgon, Vie quotidienne, p. 312-314, pensant à Pyrgi et à son sanctuaire de Leucothée, $\mathrm{M}$. TORELLI, in ArchClass, 20, 1968, p. 228, à Santa Marinella.

20 Voir art. cit. n. 3, ainsi que J. CHAMPEAUX, Oracles institutionnels et formes populaires de la divination italique, in La divination dans le monde étruscoitalique, II, Caesarodunum, Suppl. 54 (1986), p. 90-113.

21 Pour le détail des exemples, on se reportera à l'article de A. MAGGIANI. Sur Suris et Aplu, voir maintenant G. ColonA, Notivà sui culti di Pyrgi, in RPAA, 57 (1984-1985), p. 57-88.

22 Voir n. 3. A. MAGGIANI, se fondant sur les représentations figurées où apparaît la tête d'Orphée, admet «l'existence d'un genre de réponses oraculaires liées à des cérémonies nuptiales» (p. 29), mais tout en reconnaissant qu'elles n'existent sans doute plus à époque historique. Nous serions pour notre part porté à attribuer une valeur purement mythique au thème. 
La référence aux prophètes dans les légendes nationales ne débouche donc nullement, dans la réalité de l'Étrurie historique, sur le recours à une révélation intérieure. La pratique mantique étrusque est tout autre. C'est en ce sens qu'on peut parler d'un paradoxe, le paradoxe étrusque : une parole prophétique sans conséquence sur les formes effectives du contact avec la divinité, pourtant si essentiel aux yeux de ce peuple.

Et le paradoxe est d'autant plus flagrant que ce qu'ont révélé à leur peuple les prophètes étrusques est justement outre un ensemble de rites une série de techniques divinatoires, et spécialement les principes de l'hépatoscopie et de la brontoscopie. La parole prophétique des êtres mythiques du temps des origines révèle aux hommes le moyen de communiquer avec les dieux et pose les bases d'une mantique. Mais cette communication, par la suite, ne se fait plus par la parole insufflée par le dieu à un prophète : elle se ramène à l'observation et à l'interprétation de signes.

Mais c'est qu'il faut préciser un point en ce qui concerne les prophètes étrusques. Si on met à part les figures qui n'appartiennent pas vraiment à la réalité nationale, comme $\mathrm{Cacu}^{23}$ ou Orphée, on constate qu'elles sont à la base, en fait, non d'une révélation orale, mais d'une révélation écrite. On a pu définir la religion étrusque, autant comme une "religion du livre» que comme une religion de la parole ${ }^{24}$. Ce qu'auraient dit Tagès ou Vegoia apparaît en fait sous une forme écrite, leur parole ayant été soigneusement consignée par écrit par ceux qui l'ont entendue, en particulier Tarchon, le héros fondateur de Tarquinia, dans le premier cas ${ }^{25}$, Arruns Velthymnus dans le second. Désormais ce qui existe, ce n'est plus la parole d'un prophète, mais un ensemble d'écrits, ces libri qui renfermaient la science religieuse toscane, qui avaient tellement frappé les Romains qui, pas plus que les Grecs, n'en possédaient l'équivalent, et dont à sa manière un critique comme Lucrèce témoigne du succès ${ }^{26}$.

La parole prophétique se transmue donc dans les faits dans la fixité d'un écrit qui est censé la conserver, ne varietur, pour les générations

23 Voir n. 3. Cacu n'a pas été à la source de libri étrusques, mais on voit sur les représentations figurées un petit personnage, Artile, mettant soigneusement par écrit - tout comme les auditeurs de Vegoia ou Tagès - les dires du prophète.

24 Voir dans ce sens aussi les auteurs cités aux n. 1 et 2.

25 C'est le cas dans la version de I. LYD., De Ost., pr., 2-3. Sur la question de l'interlocuteur de Tagès (qui est un bubulcus anonyme en CrC., De div., II, 23, 50), voir notre ouvrage $L$ 'origine lydienne des Étrusques, à paraître, Rome.

26 Voir VI, 381-382 : non Tyrrhena retro volventem carmina frustra/indicia occultae divum perquirere mentis. Sur ce passage, J. HEURGON, Vie quotidienne, p. 278. 
futures, à partir du lointain temps des origines où elle aurait été mise par écrit 27 . Cicéron, par exemple, le précise bien pour Tagès : ses auditeurs ont noté toutes ses paroles, et c'est de cela qu'est née l'Etrusca disciplina ${ }^{28}$. La forme sous laquelle se présente en pratique la parole de Tagès ou de Vegoia est celle des libri qu'on leur attribuait - et qui bien sûr ont tendu à se multiplier avec le temps ${ }^{29}$ ! Il est certain en effet qu'il était aisé de faire circuler des textes de prophéties mises sur le compte de ces êtres prestigieux, et visant un but bien précis dans les circonstances du moment : tel est clairement le sens de la prophétie de Vegoia qui nous est parvenue ${ }^{30}$. Il est significatif en tous cas que, destinées au présent, ces injonctions présentées comme d'origine divine ne s'expriment pas par la bouche d'un être se disant investi d'une mission, d'une révélation, mais prennent le détour d'un écrit, qui se dit certes fondé sur une parole, mais une parole située dans le temps lointain des origines, hors du présent où le prophétisme n'a plus cours.

Le sens que prend cette consignation par écrit dans le cadre de la société étrusque est clair. Censorinus le précise, ce sont les lucumons, les chefs des cités, qui transcrivent les paroles de Tagès 31 . Ce sont donc les représentants de l'aristocratie étrusque qui s'arrogent le droit de disposer de la parole inspirée et de veiller à ses applications. Ce qu'illustre éloquemment le fait que ces principes qui représentaient la couche dominante dans les cités aient aimé se faire représenter sur leurs tombeaux, avec à leurs côtés ces libri où était exposé le contenu de

27 Pour les Étrusques, l'origine de la science religieuse nationale se confond avec les origines du peuple lui-même. Tarchon, qui recueille les paroles de Tagès, est le fondateur mythique de Tarquinia et de l'ensemble de la dodécapole chez STRAB., V, 2, 2 (219), reflétant une tradition locale.

28 CIC., op. cit. : tum illa plura locutum multis audientibus, qui omnia verba ejus exceperint litterisque mandarint; omnem autem orationem fuisse eam, qua haruspicinae disciplina contineretur.

29 Pour Tagès, CIC., op. cit.., parle d'haruspicine, Censorinus, De die nat., IV, 13, d'extispicine, mais ServiUs, ad VERG., Aen., VIII, 398, lui rapporte les livres des morts toscans (libri Acheruntici), I. LYD., De ost., 54; De mens., 79, des traités sur les séismes, et Nigidius Figulus lui attribuait la paternité d'un calendrier brontoscopique (I. LYD., De ost., 27-41). Pour Vegoia, SERVIUs lui rapporte des libri d'art fulguratoire, AMMIEN des libri en général (références n. 6).

30 La date de ce texte, et partant sa signification sont très discutées. Outre A. VALVo, art. cit. n. 6, voir p. ex. les propositions de J. HEURGON, The Date of Vegoia's Prophecy, in JRS, 49 (1959), p. 41-45; R. TURCAN, Encore sur la prophétie de Vegoia, in Mélanges J. Heurgon, Rome, 1976, p. 1009-1019.

31 CENs., De die nat., IV, 13 : quam lucumones tum Etruriae potentes exscripserunt. 
l'Etrusca disciplina ${ }^{32}$. Leurs familles veillaient à ce que cette science soit transmise en leur sein de génération en génération ${ }^{33}$. Et leurs haruspices - au moins les officiels, sinon les charlatans, ces haruspices vicani qui s'étaient inévitablement multipliés ! - appartenaient aux grandes familles ${ }^{34}$; l'exemple de Spurinna, haruspice de César, en est une parfaite illustration : il appartient à la puissante gens de Tarquinia qui devait étaler les éloges de ses ancêtres sur le forum de la citét ${ }^{35}$. Assurément, la liberté de la parole inspirée pouvait difficilement s'accommoder de la structure figée de la société étrusque, dominée par l'aristocratie des principes ${ }^{36}$.

Ainsi donc, valorisée par la légende, la parole inspirée se voit en pratique évacuée hors de la réalité présente. Elle appartient à un stade révolu, celui d'un mythique temps des origines, sans commune mesure avec le temps dans lequel vit présentement l'homme ${ }^{37}$.

Faut-il pour autant croire que tout sens de la parole inspirée soit exclu pour le présent chez les Étrusques, que pour eux cette forme de pratique oraculaire soit totalement rejetée hors du champ du possible? Nous ne le pensons pas. Et nous verrions la marque de cette importance qu'ils continuent à accorder à la parole inspirée dans le fait qu'elle se concrétise parfois sous nos yeux, par le recours à des formes d'oracles prophétiques : les Étrusques se sont tournés vers Delphes, ont été consulter la Pythie, et ont eu toute une politique de liens envers le grand sanctuaire panhellénique, illustrée par l'érection de deux trésors sur le site, ceux de Spina et d'Agylla-Caeré ${ }^{38}$. On voit même dans ce second cas, à travers le récit d'Hérodote narrant les péripéties de la prise de contact entre les Cérites et Delphes, après la bataille d'Alalia, le massacre des prisonniers phocéens et l'épidémie qui en fut la consé-

32 Voir F. RONCALLI, Osservazioni sui libri lintei etruschi, in RPAA, 51-52 (19781980), p. 3-22.

33 Voir CIC., Fam., VI, 6, 3, à propos de son ami Caecina. Sur un plan général, CiC., De div., I, 92; VAL. MAX., I, 1, 1, à propos de l'ordre des haruspices organisé par Rome pour son usage, et la version de la légende de Tagès de Comm. Bern. ad LUC., I, 636, où il enseigne non aux principes étrusques, mais à leurs fils.

34 Voir Cic., VAL. MAX., op. cit., parlant de principes.

35 Voir n. 17.

36 Sur ce point, p. ex. J. HEURGoN, Vie quotidienne, p. 57-73.

37 Sur cette distinction fondamentale, voir p. ex. M. ELIADE, Traité d'histoire des religions, Paris, 1970, p. 326-343.

38 Voir notre ouvrage Les Pélasges en Italie, Rome, 1984, p. 18-22, 217-221. Sur un plan plus général, M. GRAs, Trafics tyrrhéniens archaïques, Rome, 1985, p. 681693, et notre article Le città etrusche e Delfi, communication au V Convegno della Fondazione per il Museo C. Faina, Orvieto, 1988, à paraitre dans les Actes. 
quence $^{39}$, les Étrusques venir demander à la Pythie de confirmer, par sa parole sacrée, des mesures de purification prises sans doute, comme l'a justement suggéré récemment J.-P. Thuillier, en fonction des règles des libri de l'Etrusca disciplina ${ }^{40}$. Certes, les implications politiques de cette démarche étrusque sont évidentes : elle n'en traduit pas moins, ce qui nous intéresse ici, une conscience persistante de la valeur de la parole inspirée, dans le sens même, si on suit J.-P. Thuillier dans son analyse, de sa supériorité par rapport aux règles écrites des libri. Mais il est significatif que ce recours se fasse auprès d'un oracle étranger, grec, et ne se traduise pas - du moins autant que nous le sachions -, par l'introduction de pratiques analogues sur le sol toscan. Certes la parole inspirée fascine les Étrusques - on le voit aussi avec le succès du motif de la tête vaticinante d'Orphée dans leur art ${ }^{41}$-, mais elle n'en reste pas moins en marge de leurs pratiques effectives.

École Normale Supérieure

Dominique BRIQUEL

Rue d'Ulm, 45

F - 75230 PARIS

39 HDT., I, 167.

40 Dans Les conséquences de la bataille d'Alalia, oracle delphique et divination étrusque, in La divination dans le monde étrusco-italique, Caesarodunum, Suppl. 52 (1985), p. 23-32.

41 Sur ce point, A. MAGGIANI, art. cit. n. 3. 